\title{
Editor's note: Special issue on internet of things system technologies based on quality of experience
}

Published online: 7 February 2020

(C) Springer Science+Business Media, LLC, part of Springer Nature 2020

Peer-to-Peer Networking and Applications gratefully acknowledges the editorial work of the scholars listed below on the special issue entitled "Internet of Things System Technologies based on Quality of Experience":

Dr. Cho Jaeik, IBM, Korea

Dr. Naveen Chilamkurti, La Trobe University, Australia

Dr. SJ Wang, Central Police University, Taiwan

The 9 papers in this issue include:

"A scheme of Flexible and Reversible Information-hiding in SMVQ Mechanism" by Cheng-Ta Huang.

"New Efficient M2C and M2M Mutual Authentication Protocols for IoT-based Healthcare Applications" by Fatma Merabet, Amina Cherif, Malika Belkadi, Olivier Blazy, Emmanuel Conchon, and Damien Sauveron.

"Internet of Things System Technologies based on Quality of Experience" by Dongsik Son, Seungpyo Huh, Taejin Lee, and Jin Kwak.

"Weaknesses of Some Lightweight Blockciphers Suitable for IoT Systems and Their Applications in Hash Modes" by
Hangi Kim, Myungseo Park, Jaehyung Cho, Jihun Kim, and Jongsung Kim.

"DWT-based Reversible Information Hiding Scheme Using prediction-error-expansion in Multimedia Images" by Chi-Yao Weng.

"Interactive Job Sequencing System for Small Make-ToOrder Manufacturers under Smart Manufacturing Environment" by Jun Woo Kim and Soo Kyun Kim.

"Optimized Clustering in Vehicular ad hoc Networks based on Honey bee and Genetic Algorithm for Internet of Things" by Masood Ahmad, Ata UI Aziz, Ishtiaq Wahid, Fasee Ullah, Awais Ahmad, and Fakhri Alam.

"Energy and Time Efficient Task Offloading and Resource Allocation on the Generic IoT-Fog-Cloud Architecture" by Huaiying Sun, Huiqun Yu, Guisheng Fan, and Liqiong Chen.

"Forensic Analysis for IoT Fitness Trackers and its Application" by Serim Kang, Soram Kim, and Jongsung Kim. 